\title{
Perfil cognitivo de la dependencia emocional en estudiantes universitarios en Medellín, Colombia*
}

\author{
Cognitive profile of emotional dependence in \\ University Students from Medellín, Colombia
}

Recibido: junio 8 de 2010 | Revisado: septiembre 7 de 2010 | Aceptado: marzo 25 de 2011

\author{
MARIANTONIA LEMOS HoYOS** \\ CRISTINA JALLER JARAMILLO \\ Ana María González CAlle \\ Zoraya TATIANA Díaz LEÓN \\ DANiEla De la Ossa \\ Universidad CES, Medellín, Colombia
}

RES UMEN

El objetivo de este estudio fue la identificación del perfil cognitivo de los estudiantes universitarios con dependencia emocional. Ésta se plantea como un patrón orientado al otro como fuente de satisfacción y seguridad personal, donde el perfil cognitivo les llevaría a interpretar los hechos de manera diferente, basándose en cubrir sus necesidades emocionales insatisfechas. Se tomó una muestra aleatoria estratificada de 569 estudiantes universitarios. Los resultados arrojaron un modelo que explica el $89.3 \%$ de la variabilidad, con alta capacidad predictiva (sensibilidad $91.4 \%$, especificidad $97.7 \%$ ), conformado por el esquema de desconfianza/abuso, creencias centrales del trastorno de la personalidad paranoide y dependiente, distorsión cognitiva de falacia de cambio y estrategia hipodesarrollada de afrontamiento de autonomía.

Palabras clave autores

Dependencia emocional, perfil cognitivo, vulnerabilidad cognitiva, esquemas, población colombiana.

Palabras clave descriptores

Dependencia, personalidad, conducta dependiente.

\section{A B S T R A C T}

The objective of this study was to identify the cognitive profile of university students with emotional dependency. This is considered as a pattern oriented to another person as a source of satisfaction and personal safety, where the cognitive profile will make them to interpret the facts in a different way in order to cover their unsatisfied emotional needs. We use a random sample of 569 university students. The results showed a characteristic profile which explains $89.3 \%$ of the variability and a high predictive model capacity (sensitivity $91.4 \%$ and specificity $97.7 \%$ ), formed by the schema of mistrust/abuse, the cores beliefs of paranoid and dependent personality disorders, fallacy of change as cognitive distortion and underdeveloped autonomy as a coping skill.

Key words authors

Cognitive profile, emotional dependency, cognitive vulnerability, schemas, Colombian population.

Key words plus

Dependence, personality, dependent behavior. 


\section{Introducción}

La dependencia emocional se plantea como un patrón que involucra aspectos cognitivos, emocionales, motivacionales y comportamentales orientados al otro como fuente de satisfacción y seguridad personal, que implica creencias erróneas acerca del amor, de la vida en pareja y de sí mismo. Creencias que llevan a pensar, a quienes presentan este patrón, que la vida solo puede concebirse dentro de una relación, aun cuando esta les genere dolor e insatisfacción, dado que no puede colmar sus necesidades emocionales irresueltas o les produce sentimientos de inseguridad frente a la disponibilidad de una persona particular cuando la requieran (Castelló, 2005; Hirschfeld et al., 1977; Lemos \& Londoño, 2006; Norwood, 1985; Schaeffer, 1998).

Este patrón ha sido entendido en la comunidad científica como un síntoma asociado a trastornos del eje I (Davila, Steinberg, Kachadourian, Cobb \& Fincham, 2004; Loas, Borgne \& Delahousse, 1994; Robins, Bagby, Rector, Lynch \& Kennedy, 1997; Sanathara, Gardner, Prescott \& Kendler, 2003; Turner \& Turner, 1999), como una característica de algunos trastornos del eje II (Bornstein, 1998; Morse, Robins \& Gittes-Fox, 2002) y como un factor explicativo de la violencia intrafamiliar (Castelló, 2002, 2005); sin embargo, no se han encontrado estudios en torno al perfil cognitivo de quienes lo presentan.

Es posible pensar que las personas con dependencia emocional poseen un perfil distintivo por el cual interpretan los hechos de manera diferente, orientados a cubrir sus necesidades emocionales insatisfechas (Castelló, 2005; Lemos \& Londoño, 2006). Desde esta perspectiva, resulta pertinente identificar los esquemas, procesos cognitivos y estrategias de afrontamiento que discriminan su procesamiento de información, con el fin de encontrar su lugar dentro de la cadena causal de las múltiples problemáticas que con ella se asocian (Bagby-Stone, 2005), así como la orientación más eficaz de las metas terapéuticas (Caro, 1997).

La importancia de la dependencia emocional como factor de vulnerabilidad de los trastornos, no niega una necesidad normal que tenemos co- mo seres humanos de estar en contacto con otros. Autores como Bornstein (1993) han explicado ampliamente esta diferencia, y diversas investigaciones señalan que una dependencia patológica puede ser factor de riesgo para trastornos como la depresión, mientras que una necesidad normal está relacionada con calidez y valoración de las relaciones interpersonales (Castelló, 2005; Dunkley, Blankstein, Zuroff, Lecces \& Hui, 2006). Por esto, la dependencia emocional es abordada desde un modelo dimensional que la identifica como un constructo similar al de la personalidad (Deary, Peter, Austin \& Gibson, 1998; Millon \& Davis, 1998; Millon \& Everly, 1985; Tirapu, Pérez, Calvo \& Mata, 2005), en tanto su manifestación dentro de unos límites adecuados es evidencia de salud mental, mientras su activación excesiva puede ser considerada como un factor de vulnerabilidad para el desarrollo de psicopatologías.

Desde el modelo cognitivo, es claro que en cada sujeto existen ciertas creencias y estrategias que forman parte de su perfil característico (Beck et al., 2007). Estas son importantes en la medida que guían su procesamiento de la información y la formación de representaciones mentales de sí mismo y del entorno, lo que le permite al sujeto adaptarse al medio (Beck, 2000).

En este modelo cobran importancia los esquemas, estructuras funcionales de representaciones de conocimiento y de la experiencia anterior, que guían la búsqueda, codificación, organización, almacenamiento y recuperación de la información (Beck, 1987; Caro, 1997), ya que se asume que la actividad primaria del sistema de procesamiento de la información es la asignación de significados, que influyen sobre las emociones y comportamientos de las personas, y lleva a que sus sentimientos no estén determinados por las situaciones, sino por la forma como interpretan estas situaciones. Estos esquemas se desarrollan a partir de la infancia y se refieren a aspectos relacionados con el sí mismo, los otros y el futuro (Beck, 2000).

Entre estos esquemas se encuentran los desadaptativos tempranos, que se establecen a partir de los primeros lazos afectivos (Young, 1999) y tienden a perpetuarse a partir de la elección de figuras de 
apego coherentes con las expectativas generadas frente a la relación con los otros, consigo mismo y el mundo circundante. Para Young, estos esquemas se agrupan en áreas de dominio según compartan características similares, ya sea por su origen, desarrollo o mantenimiento. Cuando una persona no desarrolla adecuadamente alguna de estas áreas, presentará dificultades en el funcionamiento de por lo menos una o más áreas de dominio.

Por su parte, Beck (2000) hace referencia a las creencias centrales, las cuales define como las ideas fundamentales y profundas acerca de uno mismo, de los otros y del mundo, que se desarrollan generalmente durante la infancia y son consideradas por el individuo como verdades absolutas. Las creencias centrales negativas se caracterizan por ser rígidas, globales y generalizadas.

Además de los esquemas, el modelo cognitivo hace hincapié en los procesos, que mantienen la validez de las creencias de los pacientes a pesar de la evidencia contraria (Beck, 2000). En estos se encuentran las distorsiones cognitivas, errores del pensamiento habituales y repetitivos, que dan lugar a los productos cognitivos ilógicos y equivocados (Riso, 2006), y que conducen a descartar información ambiental en aras del mantenimiento de los esquemas cognitivos de base (Caro, 1997).

Por último, es importante nombrar la existencia de estrategias de afrontamiento, esfuerzos cognitivos y conductuales que se desarrollan para manejar las demandas específicas externas y/o internas, evaluadas como excedentes o desbordantes de los recursos del individuo (Lazarus \& Folkman, 1987). Cada sujeto tiende a utilizar las estrategias que domina, por aprendizaje o por hallazgo fortuito, en una situación de emergencia y puede configurar un estilo de afrontamiento.

La identificación de los esquemas desadaptativos tempranos, las creencias centrales, distorsiones cognitivas y estrategias de afrontamiento, permiten entonces la comprensión de la dependencia emocional desde cada uno de los elementos constitutivos del modo cognitivo que este patrón representa. Teniendo en cuenta lo anterior, esta investigación se propuso identificar el perfil cognitivo en estudiantes universitarios con depen- dencia emocional, para brindar elementos que ayuden a entender la vulnerabilidad que estos individuos poseen hacia el desarrollo de múltiples trastornos, cuando se enfrentan a acontecimientos estresantes como una ruptura afectiva (Hankin \& Abela, 2005).

\section{Método}

\section{Población y muestra}

La población de referencia estuvo constituida por estudiantes de una universidad de la ciudad de Medellín (Colombia). Se llevó a cabo un muestreo aleatorio sistemático, por facultades y semestres. El tamaño de la muestra conformada por 569 participantes se obtuvo a partir de una prevalencia de dependencia emocional del $10 \%$ y un error del $5 \%$, obtenida de datos españoles sobre la misma (La Nueva España, 2006).

La muestra estuvo conformada por estudiantes con una edad promedio de 19.89 (DE 2.43) años, con un rango de edad entre los 16 y 31 años. De esta hicieron parte 386 mujeres $(67.84 \%)$ y 183 hombres (32.16\%). Con respecto al estado civil, 558 (98.59\%) de los estudiantes eran solteros y 7 (1.24 \%) estaban casados; 331 (59.11\%) estudiantes pertenecían al estrato socioeconómico alto y 200 (35.71 \%) pertenecían al medio. Además, 111 (19.51 \%) pertenecían a la Facultad de Medicina y el menor número a la Facultad de Biología, con 40 estudiantes (7.03\%). Por último, 290 (51.15 \%) estudiantes tenía una relación de pareja, frente a $276(48.68 \%)$ que no la tenían (Tabla 1).

\section{Instrumentos}

\section{Cuestionario de Dependencia Emocional (CDE)}

Construido y validado por Lemos y Londoño (2006) con 23 ítems, alto índice de fiabilidad (alfa de Cronbach de 0.927) y una explicación de la varianza de $64.7 \%$. El instrumento evalúa 6 factores de la dependencia emocional, con alfas de Cronbach entre 0.62 y 0.87 . 
TABLA 1

Estadísticos descriptivos de las variables demográficas en 569 estudiantes universitarios

\begin{tabular}{|c|c|c|c|}
\hline \multicolumn{2}{|c|}{ Datos Demograficos } & \multirow{2}{*}{$\begin{array}{c}\mathrm{N} \\
386\end{array}$} & \multirow{2}{*}{$\begin{array}{c}\% \\
67.84\end{array}$} \\
\hline Soyo & Femenino & & \\
\hline sexo & Masculino & 183 & 32.16 \\
\hline Estado & Soltero & 558 & 98.59 \\
\hline Civil & Casado & 7 & 1.24 \\
\hline \multirow{3}{*}{ Estrato Socioeconomico } & Bajo & 29 & 5.18 \\
\hline & Medio & 200 & 35.71 \\
\hline & Alto & 331 & 59.11 \\
\hline \multirow{7}{*}{ Facultad } & Biología & 40 & 7.03 \\
\hline & Derecho & 61 & 10.72 \\
\hline & Fisioterapia & 84 & 14.76 \\
\hline & Medicina & 111 & 19.51 \\
\hline & Veterinaria & 98 & 17.22 \\
\hline & Odontología & 95 & 16.7 \\
\hline & Psicología & 80 & 14.06 \\
\hline \multirow{11}{*}{ Semestre } & 1 & 165 & 29 \\
\hline & 2 & 42 & 7.38 \\
\hline & 3 & 105 & 18.45 \\
\hline & 4 & 26 & 4.57 \\
\hline & 5 & 80 & 14.06 \\
\hline & 6 & 30 & 5.27 \\
\hline & 7 & 49 & 8.61 \\
\hline & 8 & 7 & 1.23 \\
\hline & 9 & 44 & 7.73 \\
\hline & 10 & 9 & 1.58 \\
\hline & 11 & 12 & 2.11 \\
\hline \multirow{2}{*}{ Pareja } & No & 276 & 48.68 \\
\hline & Sí & 290 & 51.15 \\
\hline
\end{tabular}

Fuente: elaboración propia.

Cuestionario de Esquemas Desadaptativos

Tempranos (Young Schema Questionnaire Long

Form - Second Edition [YSQ-L2], 1990)

Se utilizó el Cuestionario de Esquemas de Young, YSQ-L2 con propiedades psicométricas para la población Colombiana, validado por Castrillón et al (2005), el cual es una adaptación del cuestionario original desarrollado por Young y Brown (1994). La prueba consta de 45 ítems y evalúa 11 esquemas desadaptativos tempranos. Este cuestionario posee un alfa de Cronbach de 0.9093 para la prueba total y de los factores entre el 0.71 y el 0.85 .
Cuestionario de Creencias Centrales de los Trastornos de la Personalidad (CCE-TP)

Instrumento construido y validado por Londoño et al. (2007), a partir de la teoría de Beck et al. (1995), sobre los trastornos de la personalidad. La prueba evalúa 14 factores que representan el $61.3 \%$ de la varianza. La fiabilidad fue de 0.931 en total y los factores presentaron alfas de Cronbach entre 0.685 y 0.851 . 
Inventario de Pensamientos Automáticos (IPA)

Escala elaborada por Ruiz y Luján (1991), para identificar los principales tipos de pensamientos automáticos agrupados en sus 15 distorsiones correspondientes. Una puntuación de dos o más para cada pensamiento automático, suele indicar que ese tema está afectando actualmente a la persona, de manera importante. Una puntuación de seis o más en el total de cada distorsión, puede ser indicativa de que tiene cierta tendencia a incomodarse por determinada forma de interpretar los hechos de la vida

Cuestionario de Estrategias de

Afrontamiento Modificado

Basado en la Escala Coping de Lazarus y Folkman (1987), modificada por Londoño et al. (2006). La prueba consta de 69 ítems, tiene un alfa de Cronbach de 0.847 y 12 factores que representan el $58 \%$ de la varianza, con niveles de fiabilidad entre 0.651 y 0.905 .

\section{Procedimiento}

Luego de la firma del consentimiento informado, los participantes respondieron los cuesionarios de esta investigación, en una sesión que tuvo un tiempo de duración promedio de 45 minutos. No se realizó contrabalanceo en la aplicación de los instrumentos.

\section{Análisis estadístico}

La base de datos se digitó en Excel 97-2003 y se importó a SPSS 15 para su análisis estadístico. Con base en la puntuación obtenida en el CDE, se dividió la población en dos grupos, tomando como punto de referencia el percentil 75. Con posterioridad, se llevó a cabo el análisis de regresión logística para buscar los elementos del perfil cognitivo que mejor lograban explicar la presencia de dependencia emocional en estudiantes universitarios. Este análisis se llevó a cabo con todas las variables del perfil, teniendo presente que, según el paradigma del procesamiento de la información, los condi- cionales (en este caso, las distorsiones cognitivas y creencias centrales) y las conclusiones (en este caso, estrategias de afrontamiento) se desprenden del núcleo central (esquemas maladaptativos tempranos).

\section{Resultados}

\section{Prevalencia de dependencia emocional}

Los estudiantes obtuvieron puntuaciones entre 23 y 138 , con una media de $58.03(D E=21.4)$ puntos. Al dividir la muestra según el percentil 75 , se encontró que la dependencia emocional se presenta en $24.6 \%$ de los estudiantes evaluados, con una proporción de $74.6 \%$ en mujeres. No se encontró asociación entre el sexo y la dependencia emocional $(p=0.060)$.

\section{Análisis de regresión logistica}

Para establecer el perfil cognitivo que explica la presencia de dependencia emocional en estudiantes universitarios, se llevó a cabo un análisis de regresión logística, mediante el cual se llegó a un modelo que explica el $89.3 \%$ de la variabilidad, con una alta capacidad predictiva (sensibilidad $91.4 \%$ y especificidad $97.7 \%$ ). En el modelo se encuentran el esquema desadaptativo temprano de "desconfianza/abuso", las "creencias centrales del trastorno de personalidad por dependencia" y "creencias centrales del trastorno de personalidad paranoide", la distorsión cognitiva de "falacia de cambio" y la estrategia de afrontamiento (de coeficiente negativo) de "autonomía" (Tabla 2).

\section{Discusión}

La dependencia emocional se entiende como un patrón crónico de demandas afectivas insatisfechas, que llevan a la persona a buscar desesperadamente satisfacerlas mediante relaciones interpersonales estrechas (Castelló, 2002, 2005). Implica pensamientos acerca de la visión de sí mismo y de uno en relación con otros, creencias acerca del valor que tiene para el sujeto la amistad, intimidad e interdependencia; sensaciones positivas como la cercanía, 
TABLA 2

Resultados del análisis de regresión logística para la dependencia emocional, en una muestra de 569 estudiantes universitarios

\begin{tabular}{lcccc}
\hline & Coeficiente & Error estandar & Chi Cuadrado & Nivel significancia \\
\hline Desconfianza/abuso & 1.088 & 0.155 & 49.599 & $<0.001$ \\
Paranoide & 0.38 & 0.082 & 21.624 & $<0.001$ \\
Dependiente & 0.153 & 0.064 & 5.784 & 0.016 \\
Falacia de cambio & 0.519 & 0.172 & 9.073 & 0.003 \\
Autonomía & -0.482 & 0.156 & 9.552 & 0.002 \\
Constante & -26.423 & 3.74 & 49.912 & $<0.001$ \\
\hline
\end{tabular}

Fuente: elaboración propia.

afiliación, y emociones negativas generadas por la soledad y la separación; además de comportamientos orientados a mantener la cercanía interpersonal, tales como ayudar a otros, dar o pedir consejo (Hirschfeld et al., 1977).

Los resultados de esta investigación señalan que las personas con dependencia emocional presentan un perfil cognitivo caracterizado por un esquema desadaptativo temprano de "desconfianza/abuso", creencias centrales "paranoides" y "dependientes", una distorsión cognitiva de "falacia de cambio" y "autonomía" como estrategia hipodesarrollada de afrontamiento.

Según Castelló (2005), la historia de experiencias infantiles se caracteriza por situaciones lo suficientemente adversas como para generar disfuncionalidades en sus autoesquemas y pautas de interacción. A su vez, Young (1999) plantea que el estilo típico de las familias que lleva a que uno de sus miembros desarrolle el esquema de desconfianza/abuso, se caracteriza por frialdad, negligencia, abuso, soledad, impredicción, conductas explosivas y desapego, lo que trae por consecuencia una percepción del entorno como hostil, en el que el daño es percibido como intencional o como consecuencia de una injustificada y extrema negligencia, generando la sensación de que siempre se obtiene la peor parte o que siempre se termina siendo víctima de humillaciones, engaños, abusos o mentiras.

Esta sensación llevaría a estas personas a percibir las relaciones como impredecibles y mostrar comportamientos de prevención (Castelló, 2002, 2005), evidencia además de un apego ansioso am- bivalente (Norwood, 1985), explicando así lo encontrado por West, Spreng, Casares-Knight, Rose y Leiper (1998), en una muestra de psicólogos del Reino Unido en los que se encontró que aquellos con un apego ansioso ambivalente no veían a sus parejas como figuras disponibles, por lo cual experimentaban ansiedad constante de ver satisfechas sus necesidades de seguridad.

Esto concuerda con la creencia central del trastorno de personalidad paranoide encontrada en este estudio, la cual hace referencia a pensarse como una persona desconfiada y suspicaz, que se manifiesta en diversos comportamientos, como estar completamente en guardia, ser celosos y, además, estar alerta con el fin de evitar la manipulación de los otros (Londoño et al., 2007).

Además se relaciona con los hallazgos de Bornstein, Geiselman, Eisenhart y Languirand (2002), quienes evaluaron un grupo de estudiantes universitarios para estudiar el perfil relacional, afirmando que aquellos con alta sobredependencia destructiva experimentaban ansiedad con relación a la cercanía, intimidad y disponibilidad de sus cuidadores, sumada a extrema preocupacion por el posible abandono; comportamientos que pueden ser comprendidos desde el temor a la soledad, la ansiedad por separación y la búsqueda de expresión afectiva, los cuales son característicos de la dependencia emocional (Lemos \& Londoño, 2006).

Sin embargo, esto se hace más complejo a la luz de la creencia del trastorno de personalidad por dependencia, que lleva a estas personas a considerarse débiles, necesitados de ayuda y afecto, y a los 
otros como fuentes de seguridad (Londoño et al., 2007), lo que corrobora lo reportado por Castelló (2005) quien afirma que las personas con dependencia emocional se sienten desvalidas, necesitadas y débiles afectivamente, razón por la cual tratan de unirse a una figura más fuerte que les proporcione protección. Además esto es concordante con lo reportado por Morse et al. (2002) acerca de la correlación entre sociotropía y trastorno de personalidad por dependencia, al evaluar una muestra de pacientes psiquiátricos.

Los sentimientos de inferioridad y baja autoconfianza, manifestaciones de esta creencia, han sido reportados en poblaciones universitarias con altos niveles de sobredependencia destructiva (Bornstein et al., 2004), así como en poblaciones clínicas con altos niveles de sociotropía, por Robins et al. (1997). Esto explicaría también la presencia de sintomas autopunitivos, característicos de los pacientes depresivos con alta sociotropía (Burke \& Haslam, 2001).

Entre los comportamientos que evidencian esta creencia están la búsqueda de atención y la expresión límite, que tienen como objetivo la contención de la pareja dentro de la relación (Lemos \& Londoño, 2006), la ansiedad por separación, la expresión afectiva de la pareja y el temor a la soledad, características que también evidencian una creencia dependiente, ya que hacen referencia al malestar que genera pensarse a sí mismo sin alguien que satisfaga aquellas necesidades afectivas irresueltas (Lemos \& Londoño, 2006) y producto, posiblemente, de un estilo de apego ansioso ambivalente presente en los dependientes emocionales (Lemos, Londoño \& Zapata, 2007; Norwood, 1985).

Siguiendo con el perfil cognitivo, para confirmar y mantener los esquemas y creencias, se encontró la distorsión cognitiva de "falacia de cambio", que puede definirse como el proceso que lleva a creer que el bienestar de uno mismo depende de manera exclusiva de los actos de los demás (Ruiz \& Imbernon, 1996), creyendo que para cubrir sus necesidades, son los otros quienes primero han de cambiar su conducta.

$\mathrm{Al}$ respecto, en el estudio realizado por Lemos et al. (2007) con una población no clínica, entre los 18 y 55 años, se encontró que las personas con dependencia emocional presentaban significativamente las distorsiones cognitivas de "deberías" y falacia de control. Se señala que "los deberías" pueden llevar a una baja calidad en la relación, debido a que se imponen reglas para mantener supuestos que permitan confirmar sus estándares acerca de las relaciones (Lemos et al., 2007), asuntos que pueden explicarse igualmente desde la falacia de cambio, encontrada en el presente estudio.

Por otro lado, la distorsión cognitiva de falacia de control refleja pensamientos de tipo "yo soy responsable del sufrimiento de los que me rodean", "si otros cambiaran su actitud yo me sentiría mejor" (Lemos et al., 2007), verbalizaciones que muestran que dicha falacia es subyacente a la falacia de cambio, ya que el dependiente emocional, al demandar constantemente de su pareja cambios que lleven a su propio bienestar y no obtenerlos, genera un sentimiento de responsabilidad frente al bienestar de su pareja, tal y como se describe en la falacia de control.

Desde esta perspectiva, es evidente que los estudiantes universitarios con dependencia emocional consideran que, para alcanzar la felicidad buscada, es necesario que sea la pareja quien cambie. Esto se manifiesta en comportamientos tales como: modificación de actividades y propósitos que tanto ellos como sus parejas deben realizar, necesidad de una constante expresión afectiva por parte de la pareja (Lemos \& Londoño, 2006) y deseo de exclusividad, lo cual los lleva a creer que tienen derecho a exigir de su pareja conductas orientadas a satisfacer únicamente sus necesidades emocionales por encima de las demás personas de su círculo social (Castelló, 2000, 2005). Lo anterior corrobora lo encontrado por Lynch, Robins y Morse (2001) en una muestra de pacientes depresivos, donde aquellos que presentaban altos niveles de sociotropia, tendían a comportarse de forma demandante con el consecuente alejamiento por parte de sus parejas.

Por último, debe resaltarse el hipodesarrollo de la estrategia de afrontamiento de autonomía, que se define como la tendencia a responder ante un problema con la búsqueda independiente las soluciones, sin contar con el apoyo de otras perso- 
nas tales como amigos, familiares o profesionales (Londoño et al., 2006). El hipodesarrollo de esta estrategia se evidencia en comportamientos de aferramiento hacia su pareja y búsqueda de atención, así como en la modificación de planes (Lemos \& Londoño, 2006), pues la vida de quien presenta este patrón gira alrededor de su pareja, hasta el punto de abandonar y modificar sus planes previos con el fin de satisfacerla, es decir, un comportamiento que se aleja notoriamente de la autonomía como tal.

En síntesis, se puede decir que el mantenimiento esquemático planteado por Young (1999) se da de una manera evidente en la dependencia emocional. Al analizar cada componente del perfil cognitivo de quienes presentan dependencia emocional, se destacan características que reflejan creencias, distorsiones y estrategias de afrontamiento que mantienen y refuerzan el esquema desadaptativo temprano predominante, perpetuándolo e inflexibilizándolo a lo largo de la vida. En el aspecto conductual, tanto la expresión límite como la modificación de planes y la búsqueda de atención son conductas autoderrotistas que, sumadas a procesos cognitivos evidentes en el temor a la soledad, la ansiedad de separación y la expresión afectiva, llevan a la confirmación y conservación del esquema.

Los resultados arrojados por la investigación permiten confirmar que la ansiedad de separación y la demanda de expresión afectiva de la pareja, características básicas de la persona con dependencia emocional, son manifestaciones comportamentales del esquema de desconfianza/abuso, en el que una posible disolución de la relación genera, en quien presenta el patrón, un temor intenso a ser traicionado, evidencia de su creencia paranoide; un aferramiento excesivo a la pareja, muestra de su creencia dependiente y demandas respecto a la expresión de afecto, ocasionadas por la distorsión cognitiva de falacia de cambio (Schaeffer, 1998). Por último, esta desconfianza a la disponibilidad afectiva de su pareja y la necesidad de contar con ella, puede llevar a quien presenta dependencia emocional, a que cuando se siente amenazado, es decir, a punto de ser abandonado, utilice estrategias como la expresión límite, que sería una evidencia del hipodesarrollo de la autonomía como estrategia de afrontamiento (Lemos \& Londoño, 2006).

\section{Referencias}

Bagby-Stone, S. (2005). Cognitive vulnerability to emotional disorders. Mahwah, NJ: Erlbaum.

Beck, A. T. (1987). Cognitive models of depression. Journal of Cognitive Psychotherapy. An International Quarterly, 1(1), 5-37.

Beck, A. T., Freeman, A., Davis, D., Pretzer, J., Fleming, B., Ottaviani, R., et al. (2007). Cognitive Therapy of Personality Disorders (2. ${ }^{\mathrm{a}}$ ed.). New York: Guilford.

Beck, A. T., Freeman, A., Davis, D., Pretzer, J., Fleming, B, Ottaviani, R., et al. (1995). Terapia cognitiva de los trastornos de la personalidad. Buenos Aires: Paidós.

Beck, J. (2000). Terapia cognitiva. Barcelona: Gedisa.

Bornstein, R. F. (1993). The dependent personality. New York: Guilford.

Bornstein, R. F. (1998). Implicit and self-attributed dependence needs in dependent and histrionic personality disorders. Journal of Personality Assessment, 71(1), 1-14.

Bornstein, R. F., Geiselman, K. J., Gallagher, H. A., Ng, H. M., Hughes, E. E. \& Languirand, M. A. (2004). Construct validity of the Relationship Profile Test: Impact of gender, gender role and gender role stereotype. Journal of Personality Assessment, 82(1), 104-113.

Bornstein, R. F., Geiselman, K. J., Eisenhart, E. A. \& Languirand, M. A. (2002). Construct validity of the Relationship Profile Test: Links with attachment, identity, relatedness, and affect. Assessment, 9(4), 373-381.

Burke, A. \& Haslam, N. (2001). Relations between personality and depressive symptoms: A multimeasure study of dependency, autonomy, and related constructs. Journal of Clinical Psychology, 57(7), 953-961.

Caro, I. (1997). Manual de psicoterapias cognitivas. Barcelona: Paidós.

Castelló, J. (2002, diciembre). Tratamiento de la dependencia emocional en la mujer. Ponencia presentada en el II Symposium Nacional de Adicción en la Mujer, Fundación Instituto Spiral, Madrid, España. Recuperado el 30 de abril de 2004, de http:// 
www.institutospiral.com/cursos $\% 20 y \% 20$ seminarios/resumenes/Jorge\%20Castelló.htm

Castelló, J. (2005). Dependencia emocional. Características y tratamiento. Madrid: Alianza Editorial.

Castrillón, D., Chávez, L., Ferrer, A., Londoño, N. H., Maestre, K., Marín, C. A., et al. (2005). Validación del Young Schema Questionnaire Long Form-Second Edition (YSQ-L2) en población colombiana. Revista Latinoamericana de Psicología, 37(3), 541-560.

Davila, J., Steinberg, S. J., Kachadourian, L., Cobb, R. \& Fincham, F. (2004). Romantic involvement and depressive symptoms in early and late adolescence: The role of a preoccupied relational style. Personal Relationships, 11(2), 161-179.

Deary, I. J., Peter, A., Austin, E. J. \& Gibson, G. J. (1998). Personality traits and personality disorders. British Journal of Psychology, 89, 647-661.

Dunkley, D. M., Blankstein, K. R., Zuroff, D. C., Lecces, S. \& Hui, D. (2006). Neediness and connectedness and the five-factor model of personality. European Journal of Personality, 20, 123-136.

Hankin, B. L. \& Abela, J. R. Z. (2005). Development of Psychopathology: A vulnerability-stress perspective. Thousand Oaks, California: Sage.

Hirschfeld, R. M. A., Klerman, G. L., Gough, H. G., Barrett, J., Korchin, S. J. \& Chodoff, P. (1977). A measure of interpersonal dependency. Journal of Personality Assessment, 41(6), 610-618.

Lazarus, R. \& Folkman, S. (1987). Estrés y procesos cognitivos. Barcelona: Martínez Roca.

Lemos, M. \& Londoño, N. H. (2006). Construcción y validación del Cuestionario de Dependencia Emocional en población colombiana. Acta Colombiana de Psicología, 9(2), 127-140.

Lemos, M., Londoño, N. H. \& Zapata, J. A. (2007). Distorsiones cognitivas en personas con dependencia emocional. Informes Psicológicos, 9, 55-69.

Loas, G., Borgne, J. Y. \& Delahousse, J. (1994). Affective dependency and primary alcoholism. Schweizer Archiv für Neurologie, Neurochirurgie und Psychiatrie, 145(4), 35-38.

Londoño, N. H., Henao, G. C., Puerta, I. C., Posada, S., Arango, D. \& Aguirre, D. C. (2006). Propiedades psicométricas y validación de la Escala de Estrategias de Coping Modificada (EEC-M) en una muestra colombiana. Universitas Psychologica, 5(2), 327-349.

Londoño, N. H., Maestre, K., Marín, C. A., Schnitter, M., Castrillón, D., Ferrer, A., et al. (2007). Validación del Cuestionario de Creencias Centrales de los Trastornos de la Personalidad (CCE-TP) en población colombiana. Avances en Psicología Latinoamericana, 25(2), 138-162.

Lynch, T. R., Robins, C. J. \& Morse, J. Q. (2001). Couple functioning in depression: The roles of sociotropy and autonomy. Journal of Clinical Psychology, 57(1), 93-103.

Millon, T. \& Everly, G. S. (1985). La personalidad y sus trastornos. Barcelona: Martínez Roca.

Millon, T. \& Davis, R. (1998). Trastornos de la personalidad: más allá del DSM-IV. Barcelona: Masson.

Morse, J. Q., Robins, C. J. \& Gittes-Fox, M. (2002). Sociotropy, autonomy, and personality disorder criteria in psychiatric patients. Journal of Personality Disorders, 16(6), 549-560.

Norwood, R. (1985). Las mujeres que aman demasiado. Madrid: Javier Vergara.

Riso, W. (2006). Terapia Cognitiva. Fundamentos teóricos y conceptualización del caso clínico. Bogotá: Norma.

Robins, C. J., Bagby, R. B., Rector, N. A., Lynch, T. R. \& Kennedy, S. H. (1997). Sociotropy, autonomy, and patterns of symptoms in patient with major depression: A comparison of dimensional and categorical approaches. Cognitive Therapy and Research, 21(3), 285-300.

Ruiz, J. J. \& Imbernon, J. J. (1996). Sentirse mejor. Como afrontar los problemas emocionales con terapia cognitiva. Psicologia Online. Recuperado el 19 de febrero de 2009, de http:/www.psicologia-online.com/ esmubeda/libros/Sentirse_Mejor/Sentirse2.htm

Ruiz, J. \& Luján, J. (1991). Manual de Psicoterapia Cognitiva. Inventario de pensamientos automáticos. Psicología Online. Recuperado el 19 de febrero de 2009, de http://www.psicologia-online.com/ESMUbeda/ Libros/SentirseMejor/sentirse2.htm

Sanathara, V. A., Gardner, C. O., Prescott, C. A. \& Kendler, K. S. (2003). Interpersonal dependence and major depression: An etiological interrelationship and gender differences. Psychological Medicine, 33(5), 927-931. 
Schaeffer, B. (1998). ¿Es amor o es adicción? Barcelona: Apóstrofe.

Tirapu, J., Pérez, A., Calvo, A. \& Mata, I. (2005). Propuesta de un modelo dimensional para los trastornos de personalidad. Actas Españolas de Psiquiatría, 33(4), 254-262.

Turner, H. A. \& Turner, R. J. (1999). Gender, social status and emotional reliance. Journal of Health and Social Behavior, 40(4), 360-73.

Young, J. \& Brown, G. (1994). Young Schema Questionnaire (2 ${ }^{\text {nd }}$. ed.). En J. E. Young (Ed.), Cognitive Therapy for Personality Disorders: A Schema Focused Approach. New York: Cognitive Therapy Center of New York.
Young, J. (1999). Terapia cognitiva para los trastornos de personalidad: una aproximación centrada en esquemas (3.. ed.). New York: Departamento de Psquiatría, Universidad de Columbia.

West, M., Spreng, S., Casares-Knight, P., Rose, S. M. \& Leiper, R. (1998). A comparison of two measures to assess adult attachment. Journal of Genetic Psychology, 159(3), 345-348. 